dynamometry group. Although the randomization was done in a strict manner, differences may still occur, and we regret that these betweengroup differences were not noted in the footnote to Table 1.

There is debate about whether baseline criteria should be incorporated into the analysis of clinical trials. When we included the presence of hypertensive disorders and diabetes in a logistic model estimating the effect of the use of a pressure catheter, we still found no significant dif- ferences in rates of instrumental delivery or neonatal outcomes between the two study groups.

Jannet J.H. Bakker, M.Sc.

Ben W. Mol, M.D.

Joris A.M. van der Post, M.D.

Academic Medical Center

Amsterdam, the Netherlands

j.j.bakker@amc.nl

Since publication of their article, the authors report no further potential conflict of interest.

\title{
Hypofractionated Radiotherapy for Breast Cancer
}

TO THE EDITOR: Whelan et al. (Feb. 11 issue) ${ }^{1}$ report 10-year results of hypofractionated radiotherapy, as compared with standard radiotherapy, in women with early breast cancer. The study showed no overall evidence of inferiority of the abbreviated 16-fraction test schedule. However, a retrospective subgroup analysis suggested that hypofractionation might be relatively less effective in patients with grade 3 tumors.

We tested this hypothesis in a meta-analysis of the UK Standardisation of Breast Radiotherapy (START) A and B trials ${ }^{2,3}$ and their pilot study. ${ }^{4}$ At 5 years, the rates of locoregional relapse were $4.9 \%$ (95\% confidence interval [CI], 4.0 to 5.9 ) in patients on the control schedule and 5.2\% $(95 \%$ CI, 4.5 to 6.0 ) in those on the combined hypofractionated schedules. A total of 4883 patients with known tumor grade were available for analysis, including 222 with locoregional relapse, with a median follow-up of 8 years. Hazard ratios for the combined hypofractionated schedules, as compared with the control schedules, were 1.28 (95\% CI, 0.87 to 1.88 ) for grade 1 and 2 tumors and 0.83 (95\% CI, 0.56 to 1.23 ) for grade 3 tumors ( $\mathrm{P}=0.12$ for interaction). Adjusted $\alpha / \beta$ values (indicating sensitivity to fraction size) were estimated as $3.6 \mathrm{~Gy}$ (95\% CI, 0 to 7.4) for grade 1 and 2 tumors and 2.2 Gy (95\% CI, 0 to 5.5) for grade 3 tumors. Our results suggest that the response to radiotherapy fraction size is not affected by tumor grade.

Joanne S. Haviland, M.Sc.

Institute of Cancer Research

Sutton, United Kingdom

jo.haviland@icr.ac.uk
John R. Yarnold, M.B., B.S.

Royal Marsden NHS Foundation Trust Sutton, United Kingdom

Søren M. Bentzen, Ph.D., D.Sc.

University of Wisconsin School of Medicine and Public Health Madison, WI

No potential conflict of interest relevant to this letter was reported.

1. Whelan TJ, Pignol J-P, Levine MN, et al. Long-term results of hypofractionated radiation therapy for breast cancer. $\mathrm{N}$ Engl J Med 2010;362:513-20.

2. The START Trialists' Group. The UK Standardisation of Breast Radiotherapy (START) Trial A of radiotherapy hypofractionation for treatment of early breast cancer: a randomised trial. Lancet Oncol 2008;9:331-41.

3. Idem. The UK Standardisation of Breast Radiotherapy (START) Trial B of radiotherapy hypofractionation for treatment of early breast cancer: a randomised trial. Lancet 2008;371:1098-107. 4. Owen JR, Ashton A, Bliss JM, et al. Effect of radiotherapy fraction size on tumour control in patients with early-stage breast cancer after local tumour excision: long-term results of a randomised trial. Lancet Oncol 2006;7:467-71. [Erratum, Lancet Oncol 2006;7:620.]

THE AUTHORS REPLY: In the meta-analysis by Haviland et al., there was no difference in outcomes between hypofractionated and conventional radiotherapy in patients with high-grade tumors. The purpose of our exploratory subgroup analysis was to examine the consistency of the treatment effect across different risk groups. Hypofractionated radiotherapy was similar to conventional radiotherapy in patients under the age of 50 years who had tumors of more than $2 \mathrm{~cm}$ in diameter that were estrogen-receptor-negative or who received systemic therapy, but it was less effective in those with high-grade tumors. Can our findings be reconciled with the results of the metaanalysis? 
Cross-study comparisons are limited. There are important differences between the U.K. and Canadian trials that could account for the discrepancy in findings (e.g., a shorter follow-up, inclusion of node-positive patients, and the use of boost radiation and anthracyclines). ${ }^{1-3}$ Furthermore, our analysis on tumor grade was post hoc and only one of five factors that we considered. The differential effect between treatments in patients with high-grade tumors requires validation through future research. Meanwhile, we believe it is premature to make any decisions concerning the avoidance of hypofractionated radiation in treating women with high-grade tumors.

Timothy J. Whelan, B.M., B.Ch.

Juravinski Cancer Centre

Hamilton, ON, Canada

tim.whelan@jcc.hhsc.ca
Mark N. Levine, M.D.

McMaster University

Hamilton, ON, Canada

Jim A. Julian, Ph.D.

Ontario Clinical Oncology Group

Hamilton, ON, Canada

Since publication of their article, the authors report no further potential conflict of interest.

1. Owen JR, Ashton A, Bliss JM, et al. Effect of radiotherapy fraction size on tumour control in patients with early-stage breast cancer after local tumour excision: long-term results of a randomised trial. Lancet Oncol 2006;7:467-71. [Erratum, Lancet Oncol 2006;7:620.]

2. The START Trialists' Group. The UK Standardisation of Breast Radiotherapy (START) Trial A of radiotherapy hypofractionation for treatment of early breast cancer: a randomised trial. Lancet Oncol 2008;9:331-41.

3. Idem. The UK Standardisation of Breast Radiotherapy (START) Trial B of radiotherapy hypofractionation for treatment of early breast cancer: a randomised trial. Lancet 2008;371:1098-107.

\section{Alzheimer's Disease}

TO THE EDITOR: In their article on Alzheimer's disease, Querfurth and LaFerla (Jan. 28 issue) ${ }^{1}$ stated that "the level of muscarinic acetylcholine receptors, or receptor coupling, is reduced in the brains of patients with Alzheimer's disease." However, a recent review indicated that not all studies reported such reductions and that they may not occur until the disease is advanced. ${ }^{2}$ Current study methods do not generally allow determination of muscarinic type 1 (M1) receptor density or receptor coupling status in specific neuronal pathways. In fact, in vivo studies suggest an apparent increased sensitivity to some of the effects of the M1 agonist arecoline in relatively early stages of Alzheimer's disease. ${ }^{3,4}$ Presynaptic M1 receptors in CA3 pyramidal neurons of the hippocampus have been shown to play a critical role in metabotropic glutamate receptor-mediated long-term depression of Schaffer collaterals. ${ }^{5}$ Thus, if confirmed, hypersensitivity of M1 receptors in Alzheimer's disease, especially in this neuronal pathway, could be an additional mechanism that contributes to synaptic failure and memory dysfunction in Alzheimer's disease.

Nunzio Pomara, M.D.

John J. Sidtis, Ph.D.

Nathan S. Kline Institute for Psychiatric Research

Orangeburg, NY

pomara@nki.rfmh.org

No potential conflict of interest relevant to this letter was reported.
1. Querfurth HW, LaFerla FM. Alzheimer's disease. N Engl J Med 2010;362:329-44.

2. Thathiah A, De Strooper B. G protein-coupled receptors, cholinergic dysfunction, and Abeta toxicity in Alzheimer's disease. Sci Signal 2009;2(93):re8.

3. Pomara N, Stanley M, LeWitt PA, Galloway M, Singh R, Deptula D. Increased CSF HVA response to arecoline challenge in Alzheimer's disease. J Neural Transm Gen Sect 1992;90:53-65.

4. Tariot PN, Cohen RM, Welkowitz JA, et al. Multiple-dose arecoline infusions in Alzheimer's disease. Arch Gen Psychiatry 1988;45:901-5.

5. Kamsler A, McHugh TJ, Gerber D, Huang SY, Tonegawa S. Presynaptic $\mathrm{m} 1$ muscarinic receptors are necessary for mGluR long-term depression in the hippocampus. Proc Natl Acad Sci U S A 2010;107:1618-23.

TO THE EDITOR: I wonder whether Querfurth and LaFerla have an opinion as to whether Alzheimer's is a single disease. Could it be that there are multiple causes of Alzheimer's "disease," some of which respond better, albeit still poorly, to some interventions than others - hence the variable progression of the syndrome? Might we be wiser to call it Alzheimer's syndrome?

Knight Steel, M.D.

Hackensack University Medical Center

Hackensack, NJ

ksteel@humed.com

No potential conflict of interest relevant to this letter was reported.

THE AUTHORS REPLY: The question of the level and function of muscarinic acetylcholine receptors 